\title{
Ultrafast Low-Temperature-Grown Epitaxial GaAs Photodetectors Transferred on Flexible Plastic Substrates
}

\author{
M. Mikulics, R. Adam, M. Marso, A. Förster, P. Kordoš, H. Lüth, S. Wu, X. Zheng, and Roman Sobolewski
}

\begin{abstract}
We demonstrate low-temperature (LT)-grown GaAs photodetectors transferred on flexible polyethylene terephthalate (PET) plastic substrates. The LT-GaAs layer was patterned into $20 \times 20 \mu \mathrm{m}^{2}$ chips, which after placing on the PET substrates were integrated with coplanar strip transmission lines. The devices exhibit low dark currents $\left(\leq 2 \times 10^{-8} \mathrm{~A}\right)$, subpicosecond photoresponse time, and signal amplitudes up to $\sim 0.9 \mathrm{~V}$ at the bias voltage of $\leq 80 \mathrm{~V}$ and under laser beam excitation power of $\leq 8 \mathrm{~mW}$ at $810-\overline{\mathrm{nm}}$ wavelength. At the highest bias $(\sim 80 \mathrm{~V})$ level, an increase of the response time (up to $1.3 \mathrm{ps}$ ) was observed and attributed to the influence of heating effects due to low thermal conductivity of PET. Our LT-GaAs-on-PET photodetectors withstand hundredfold mechanical bending of the substrate and are intended for applications in hybrid optoelectronic circuits fabricated on noncrystalline substrates, in terahertz imaging, and in biology-related current-excitation tests.
\end{abstract}

Index Terms-Flexible, liftoff technique, low-temperature-grown epitaxial GaAs (LT-GaAs), photodetector, plastic substrate.

\section{INTRODUCTION}

C URRENTLY, there is a growing interest in development of electronic devices and circuits on thin flexible plastic substrates because of their flexibility, high surface resistivity, chemical stability, optical transparency, and low substrate costs. Encouraging results have been reached at fabrication of fieldeffect transistors [1], light-emitting diodes [2], displays [3], and thin-film solar cells [4]. Other devices, like magnetic storage disks [5] and pyroelectric optical sensors [6] on flexible substrates are also under investigation. However, there is also a need for fast optical photodetectors and photoswitches on transparent plastic substrate for hybrid optoelectronic systems. Such systems have large potential in applications, ranging from

Manuscript received December 3, 2004; revised March 23, 2005.

M. Mikulics, R. Adam, M. Marso, A. Förster, P. Kordoš, and H. Lüth are with the Institute of Thin Films and Interfaces, cni-Center of Nanoelectronic Systems for Information Technology, Research Centre Jülich, Jülich D-52425, Germany (e-mail: m.mikulics@fz-juelich.de).

$\mathrm{S}$. Wu is with the Department of Electrical and Computer Engineering, the Laboratory for Laser Energetics, University of Rochester, Rochester, NY 146270231 USA, and also with the Department of Physics and Astronomy, University of Rochester, Rochester, NY 14627 USA.

$\mathrm{X}$. Zheng is with the Department of Electrical and Computer Engineering, the Laboratory for Laser Energetics, University of Rochester, Rochester, NY 14627-0231 USA.

R. Sobolewski is with the Department of Electrical and Computer Engineering, the Laboratory for Laser Energetics, University of Rochester, Rochester, NY 14627-0231 USA, and also with the Institute of Physics, Polish Academy of Sciences, Warszawa PL-02668, Poland.

Digital Object Identifier 10.1109/LPT.2005.851025

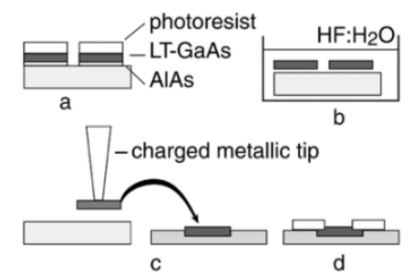

Fig. 1. Fabrication scheme of freestanding LT-GaAs photodetectors transferred on nonnative substrates using the liftoff technique.

low-cost contactless optical switching of electronic circuits to terahertz-frequency communication and biology applications. Low-temperature-grown epitaxial GaAs (LT-GaAs) photodetectors offer ultrafast photoresponse speed $(<0.5 \mathrm{ps})$, very large bandwidth $(>1 \mathrm{THz})$, and reasonable sensitivity (voltage amplitudes up to $2 \mathrm{~V}$ ) [7], [8]. In their freestanding version [8], [9], the epitaxial LT-GaAs film is lifted-off from its native GaAs substrate by selective chemical etching [10] and subsequently transferred on top of a host substrate. Polyethylene terephthalate (PET) is the plastic substrate of choice for electronic applications [1]-[6], since it is flexible, optically transparent, withstands chemical corrosion, and has large $\left(\sim 10^{13} \Omega / \mathrm{sq}\right)$ surface resistivity [11]. However, the PET substrate cannot be heated above $\sim 120{ }^{\circ} \mathrm{C}$, therefore, only LT fabrication procedures can be used. In this letter, we present ultrafast freestanding LT-GaAs photodetectors transferred on the PET substrate and integrated with coplanar strip (CPS) transmission lines. Our photodetectors exhibit nearly the same performance parameters as devices fabricated on top of crystalline substrates, such as $\mathrm{MgO}, \mathrm{SiO}_{2}$, or $\mathrm{Al}_{2} \mathrm{O}_{3}$ but the plastic substrates are flexible and low cost, and also compatible with biological systems.

\section{DEVICE FABRICATION}

Preparation of our LT-GaAs-on-PET devices consisted of the following steps: 1) molecular beam epitaxy (MBE) growth of a LT-GaAs layer; 2) separation of the LT-GaAs film from its native substrate; 3) transfer of the $20 \times 20 \mu \mathrm{m}^{2}$ size LT-GaAs chip onto the PET substrate; and 4) deposition of CPS lines connecting the photodetector and fabrication of metallic contacts. The fabrication scheme of freestanding LT-GaAs photodetectors is illustrated in Fig. 1. In the first step, a 1- $\mu$ m-thick undoped LT-GaAs epitaxial film was grown on top of a 300-nm-thick AlAs interlayer on a 2-in semi-insulating GaAs wafer by an MBE technique using the Varian GEN-II equipment. The growth process was performed at $250{ }^{\circ} \mathrm{C}$ (thermocouple reading from the substrate side), followed by in situ 


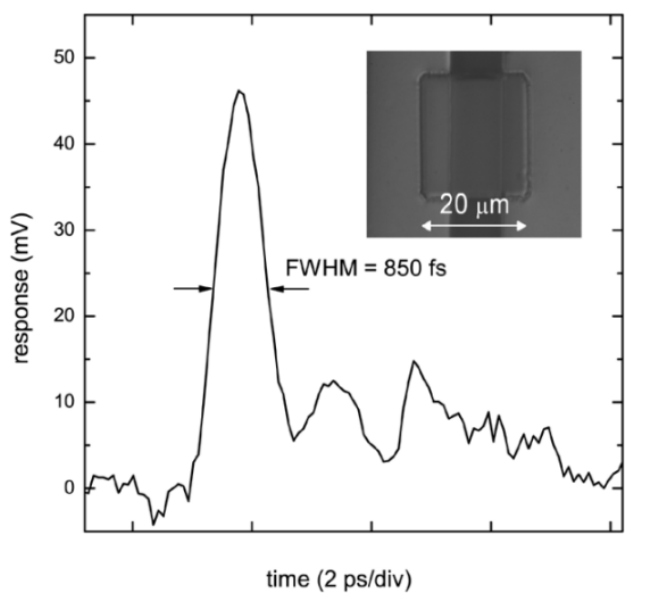

Fig. 2. Temporal response of an LT-GaAs photodetector transferred on a flexible PET substrate, and measured at $50-\mathrm{V}$ bias voltage and under $2-\mathrm{mW}$ optical input power. The inset shows a micrograph of the actual LT-GaAs chip with Ti-Au CPS lines.

isothermal annealing at $\sim 600{ }^{\circ} \mathrm{C}$ for $10 \mathrm{~min}$. Next, the wafer was patterned by optical lithography and $20 \times 20 \mu \mathrm{m}^{2} \mathrm{LT}-\mathrm{GaAs}$ chips were etched using argon ion beam [Fig. 1(a)]. No damage in the active area by the etching process step was observed. The chips were then lifted-off by selective wet etching of the AlAs sacrificial layer in a diluted HF acid [Fig. 1(b)], [9], [10]. The transfer of the micrometer-sized LT-GaAs devices onto the $130-\mu \mathrm{m}$-thick PET substrate was made by using an electrostatically charged metallic tip [Fig. 1(c)] [9]. This fabrication step did not degrade the photodetector performance, e.g., by electrostatic discharge damage. To minimize the height difference between the LT-GaAs photodetector and the substrate surface, $1-\mu \mathrm{m}$-deep wells were ion-etched at predesignated photodetector locations. Excellent adhesion of the transferred chips to the PET surface was achieved due to clean interfaces and Van der Waals forces. Finally, continuous CPS transmission lines with $10-\mu \mathrm{m}$ line separation were fabricated on top of the detector, using $\mathrm{Ti}-\mathrm{Au}$ deposition and a standard liftoff technique [Fig. 1(d)]. A micrograph of a completed LT-GaAs-on-PET photodetector is shown as an inset in Fig. 2.

\section{Measurement Results}

The current-voltage $(I-V)$ characteristics in the dark and under $850-\mathrm{nm}$ continues-wave light illumination of our LT-GaAs-on-PET photodetectors were measured immediately after the fabrication [Fig. 3]. The $I-V$ curves exhibited an ohmic behavior in the bias range from 10 up to $70 \mathrm{~V}$. Space-charge effects were not observed, which indicates an efficient suppression of the high-field region near the contacts. The dark currents were $6 \times 10^{-9} \mathrm{~A}$ at $10 \mathrm{~V}$ and $\leq 10^{-7} \mathrm{~A}$ at $100 \mathrm{~V}$. The breakdown voltage was defined as the bias point, where the current suddenly increased and destroyed the device. It was in the range of $110-130 \mathrm{~V}$, similar to what we have observed in our other freestanding LT-GaAs devices [9].

Time-resolved photoresponse characterization of our LT-GaAs photoswitches was performed using 100-fs-wide $82-\mathrm{MHz}$ repetition rate optical pulses generated by a Ti : Sapphire laser at 810-nm wavelength. Electrical transients

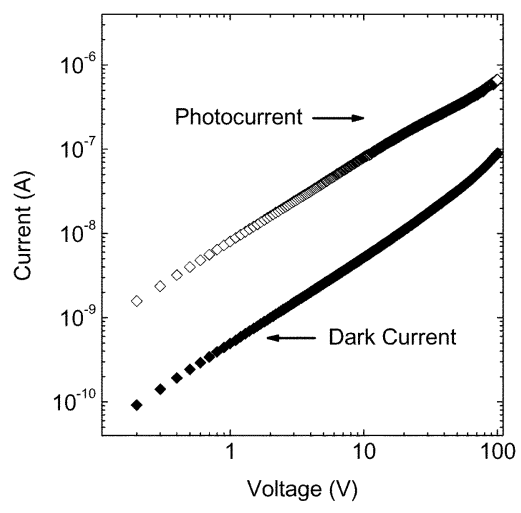

Fig. 3. $I-V$ characteristics of LT-GaAs photodetector on PET substrate. Characteristics were measured in the dark and under illumination (850-nm wavelength) with input power $100 \mu \mathrm{W}$.

were recorded with the help of an electrooptic (EO) sampling system, featuring 200-fs temporal resolution [8]. An example of the electrical transient measured at $50-\mathrm{V}$ bias and $2-\mathrm{mW}$ optical input power is shown in Fig. 2. The signal exhibits the full-width at half-maximum (FWHM) equal to 850- and 600 -fs fall time, defined as the amplitude drop to its $1 / e$ value. The obtained values are fully comparable to those reported for both the nonseparated LT-GaAs [7] (i.e., fabricated on the native GaAs substrate) and freestanding LT-GaAs devices [8], [9]. Nearly identical waveforms were recorded for other bias voltages ranging from 30 up to $100 \mathrm{~V}$, and for optical excitation powers up to $10 \mathrm{~mW}$. An approximately 10-ps-long shoulder after the main peak visible at higher bias and/or excitation power levels corresponds to the slow carrier relaxation, while the post-pulse ripples are caused by electrical signal reflections of the interfaces of our $\mathrm{LiTaO}_{3} \mathrm{EO}$ total-internal reflection probe [8].

The electrical photoresponse of our LT-GaAs-on-PET photodetectors, namely, the pulse FWHM and the pulse amplitude, plotted as functions of the incident optical power and the dc bias, are shown in Figs. 4 and 5, respectively. In Fig. 4, the data are presented for two applied bias voltages (50 and $97 \mathrm{~V}$ ), corresponding to the low- and high-bias regions. For the low-bias region $(<70 \mathrm{~V})$, the photoresponse time is essentially constant [Fig. 4(b)] and the signal amplitude increases linearly with the bias [Fig. 4(a)], as one would expect. On the other hand, in the high-bias region, while the pulse amplitude still scales linearly with the applied bias voltage, the response time increases rapidly at high optical input powers [Fig. 5]. We believe that the latter indicates reduction of the electron capture cross section with increased electric field, as it was described earlier by Zamdmer et al. [11]. We must stress, however, that at the highest bias and the optical power excitation $\left(>2.5 \mathrm{~kW} / \mathrm{cm}^{2}\right)$, heating effects must play the dominant role, due to the low thermal conductivity of the PET substrate $\left(0.15 \mathrm{Wm}^{-1} \cdot \mathrm{K}^{-1}\right)$. Device heating increases the carrier lifetime by reduction of the mobility. The influence of the device heating is also visible in Fig. 5, where we present the amplitude [Fig. 5(a)] and the response time [Fig. 5(b)] of our LT-GaAs-on-PET photodetector as a function of the applied voltage, for low $(2 \mathrm{~mW})$ and high $(10 \mathrm{~mW})$ optical input powers. We note that for the $10-\mathrm{mW}$ optical power, the pulse is wider and its FWHM increases faster at high biases, 


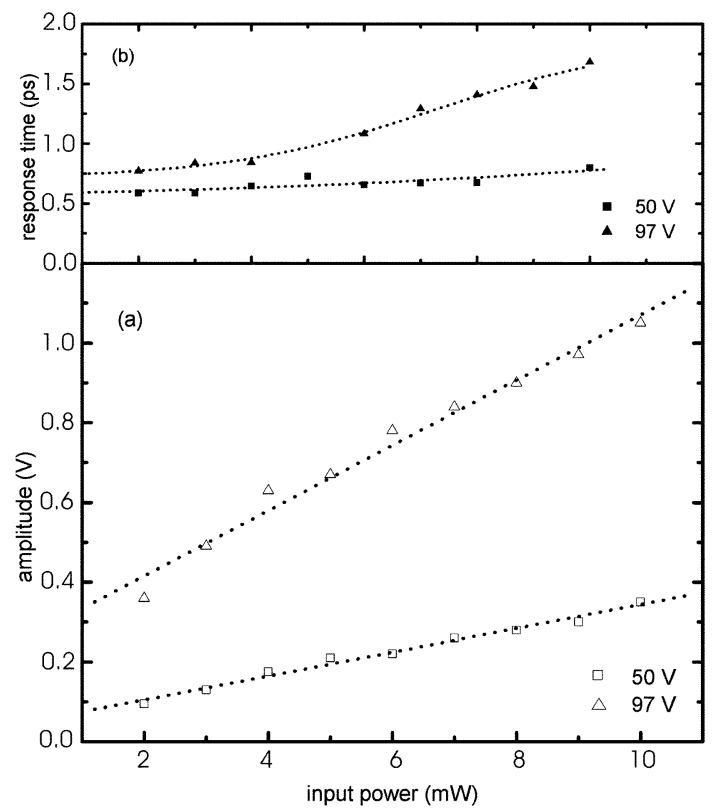

Fig. 4. (a) Amplitudes and (b) response times of the LT-GaAs-on-PET photodetector electrical transients, plotted as a function of the optical input power for the 50- and 97-V bias voltages. The dotted lines are guides to the eye.

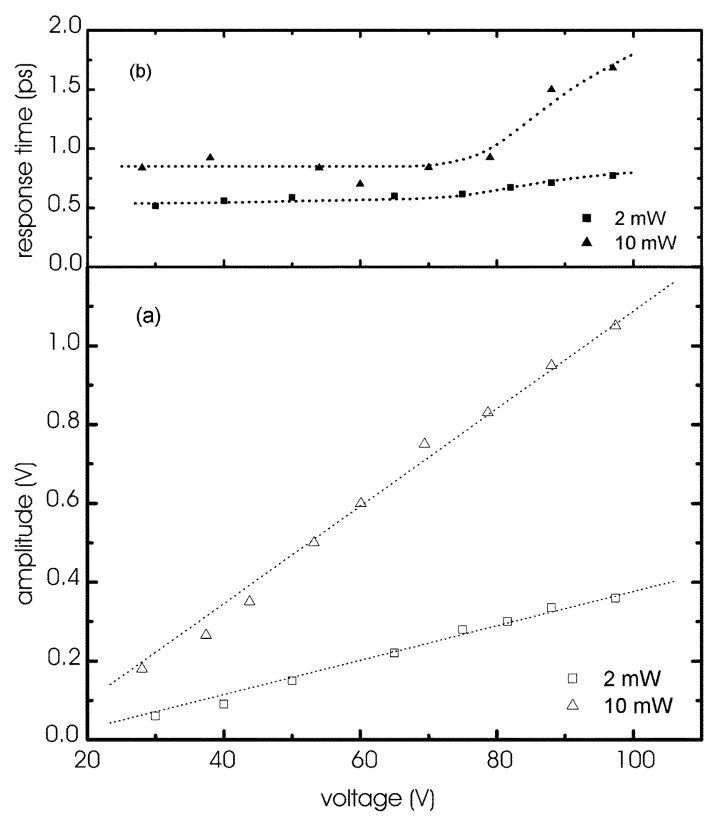

Fig. 5. (a) Amplitudes and (b) response times of the LT-GaAs-on-PET photodetector electrical transients, plotted as a function of the applied bias voltage for the 2 and $10 \mathrm{~mW}$ of the optical power excitations. The dotted lines are guides to the eye.

as compared to the $2-\mathrm{mW}$ data. The signal at the bias of $97 \mathrm{~V}$ and at $10-\mathrm{mW}$ excitation reaches the amplitude of above $1 \mathrm{~V}$ and its FWHM under these extreme conditions is very respectable with $1.3 \mathrm{ps}$.
These results were also achieved after hundredfold bending of the photodetector structure on $1.5 \times 1.5 \mathrm{~cm}^{2}$ PET substrate. The bending angle was between $90^{\circ}$ and $120^{\circ}$. No degradation of the optoelectronic properties and no destruction of the circuit was observed. Long-time measurements also did not show any degradation after $72 \mathrm{~h}$ of operation at $2-\mathrm{mW}$ optical input power and $50-\mathrm{V}$ bias voltage.

\section{CONCLUSION}

We have fabricated and tested ultrafast freestanding LT-GaAs photodetectors transferred on flexible PET substrates. Under both moderate bias and optical excitation power, our devices are characterized by the subpicosecond photoresponse time and the signal amplitude approaching $1 \mathrm{~V}$. Repetitive bending of PET substrates containing LT-GaAs devices did not lead to any measurable deterioration of the photodetector properties. The performance of our LT-GaAs-on-PET photodetectors demonstrates that they are suitable as optoelectronic devices for novel circuits on noncrystalline flexible substrates. Ultrafast photodetectors on plastic substrates should find applications for terahertz imaging and for electroexcitation of biological systems.

\section{REFERENCES}

[1] J. P. Conde, P. Alpuim, and V. Chu, "Thin-film transistors on PET at 100 degrees C," in Mater. Res. Soc. Symp. Proc., vol. 715, May 2002, pp. 679-784.

[2] R. Paetzold, K. Heuser, D. Henseler, and S. Roeger, "Performance of flexible polymetric lightemitting diodes under bending conditions," Appl. Phys. Lett., vol. 82, pp. 3342-3344, May 2003.

[3] A. B. Chwang, M. A. Rothman, S. Y. Mao, R. H. Hewitt, M. S. Weaver, J. A. Silvernail, K. Rajan, M. Hack, J. J. Brown, X. Chu, L. Moro, T. Krajewski, and N. R. Rtherford, "Thin film encapsulated flexible organic electroluminescent displays,” Appl. Phys. Lett., vol. 83, pp. 413-415, Jul. 2003.

[4] Y. Ichikawa, T. Yoshida, T. Hama, H. Sakai, and K. Harashima, "Production technology for amorphous silicon-based flexible solar cells," Solar Energy Mat. Solar Cells, vol. 66, pp. 107-115, Feb. 2001.

[5] H. Jia, J. Veldeman, and M. Burgelman, "Magnetic properties and microstructure of $\mathrm{CoCr}$ and $\mathrm{CoCrTa}$ thin films sputtered at high pressure on a PET substrate," J. Magn. Mater., vol. 223, pp. 73-80, Jan. 2001.

[6] J. Li, N. Yuan, and H. L. W. Chan, "Preparation of PCLT/P(VDF-TrFE) pyroelectric sensor based on plastic film substrate," Sens. Actuators, vol. A100, pp. 231-235, Sep. 2002.

[7] P. Kordoš, A. Förster, M. Marso, and F. Rüders, " $550 \mathrm{GHz}$ bandwidth photodetector on low-temperature grown molecular-beam epitaxial GaAs," Electron. Lett., vol. 34, pp. 119-120, Jan. 1998.

[8] X. Zheng, Y. Xu, R. Sobolewski, R. Adam, M. Mikulics, M. Siegel, and P. Kordoš, "Femtosecond response of a free-standing LT-GaAs photoconductive switch," Appl. Opt., vol. 42, pp. 1726-1731, Mar. 2003.

[9] R. Adam, M. Mikulics, A. Förster, J. Schelten, M. Siegel, P. Kordoš, $\mathrm{X}$. Zheng, S. Wu, and R. Sobolewski, "Fabrication and subpicosecond optical response of low-temperature-grown GaAs freestanding photoconductive devices," Appl. Phys. Lett., vol. 81, pp. 3485-3487, Oct. 2002.

[10] E. Yablonovich, D. M. Hwang, T. J. Gmitter, L. T. Florez, and J. P. Harbison, "Van der Waals bonding of GaAs epitaxial liftoff films onto arbitrary substrates," Appl. Phys. Lett., vol. 56, pp. 2419-2421, Jun. 1990.

[11] N. Zamdmer, Q. Hu, K. A. McIntosh, and S. Verghese, "Increase in response time of low-temperature-grown GaAs photoconductive switches at high voltage bias," Appl. Phys. Lett., vol. 75, pp. 2313-2315, Oct. 1999 . 